\title{
BMJ Open Prevalence and clustering of cardiovascular risk factors: a cross- sectional survey among Nanjing adults in China
}

Xin Hong, ${ }^{1}$ Qing Ye, ${ }^{1}$ Jing He, ${ }^{2}$ Zhiyong Wang, ${ }^{1}$ Huafeng Yang, ${ }^{1}$ Shengxiang Qi, ${ }^{1}$ Xupeng Chen, ${ }^{1}$ Chenchen Wang, ${ }^{1,2}$ Hairong Zhou, ${ }^{1,2}$ Chao Li, ${ }^{1,2}$ Zhenzhen Qin, ${ }^{1}$ Fei $\mathrm{Xu}^{1,2}$

To cite: Hong $X, Y e Q$, $\mathrm{He} \mathrm{J}$, et al. Prevalence and clustering of cardiovascular risk factors: a cross-sectional survey among Nanjing adults in China. BMJ Open 2018;8:e020530. doi:10.1136/ bmjopen-2017-020530

- Prepublication history for this paper is available online. To view these files, please visit the journal online (http://dx.doi org/10.1136/bmjopen-2017020530).

Received 8 November 2017 Revised 12 April 2018 Accepted 26 April 2018

\section{Check for updates}

1Department of Noncommunicable Disease Prevention, Nanjing Municipal Center for Disease Control and Prevention, Nanjing, China ${ }^{2}$ Department of Epidemiology and Biostatistics, School of Public Health, Nanjing Medical University, Nanjing, China

Correspondence to Professor Fei Xu; frankxufei@163.com

\section{ABSTRACT}

Objectives To estimate prevalence and clustering of cardiovascular risk factors (CRFs), and investigate the association between relevant characteristics and CRF clustering among adults in eastern China.

Design Community-based cross-sectional study. Setting Data were collected by interview survey, physical measurements and laboratory examinations from the 2011 Nanjing Chronic Disease and Risk Factor Surveillance.

Participants A representative sample of 41072 residents aged $\geq 18$ years volunteered to participate in the survey, with a response rate of $91.3 \%$. We excluded 1232 subjects due to missing data or having a history of cardiovascular diseases; a total of 39840 participants were included in the analysis.

Outcome measures Prevalence and clustering of five major CRFs including hypertension, diabetes, dyslipidaemia, overweight or obesity and current smoking.

Results Of 39840 participants (mean age $47.9 \pm 16.2$ years), 17964 (45.1\%) were men and 21876 (54.9\%) were women. The weighted prevalence of CRFs ranged between $6.2 \%$ for diabetes and $35.6 \%$ for overweight or obesity. The proportion of CRFs tended to be higher in men, the elderly, participants who lost a life partner, or lived in rural areas, or had lower level of education and total annual income. Overall, $30.1 \%$ and $35.2 \%$ of participants had one and at least two CRFs, respectively. Multivariate logistic regression revealed that men, older age, loss of a life partner, lower level of socioeconomic status, rural areas, insufficient physical activity or unhealthy diets were positively associated with CVD risk factor clustering, compared with their counterparts.

Conclusions High regional prevalence of hypertension, dyslipidaemia, overweight or obesity and their clustering are present in Nanjing. The Nanjing government should develop effective public health policies at the regional level especially for high-risk groups, such as enhancing the public's health awareness, organising health promotion programmes, implementing smoke-free law, producing healthy nutrient foods, providing free or low-cost public sports and fitness facilities.
Strengths and limitations of this study

- This is the first study designed to assess the upto-date prevalence and clustering of cardiovascular risk factors (CRFs) in Nanjing, with a large and representative adult population from eastern China $(\mathrm{n}=39$ 840).

- The high-quality study design and multistage random sampling method, the use of standard protocols and instruments, data collection by trained interviewers, a vigorous quality control throughout the survey period, a high response rate and a low percentage of missing data, ensured the validity of our self-reported data.

- A cross-sectional study cannot determine the causality or the temporal relationship of CRF clustering with the prevalence of cardiovascular disease.

- Furthermore, the information on current smoking status was based on self-report, which may be subject to reporting bias.

- The mean of the second and third blood pressure (BP) measurements in single-visit BP measurements was calculated for analysis, which may overestimate the prevalence of hypertension.

\section{INTRODUCTION}

The rapid socioeconomic progress in China has had a great impact on the lifestyle of the population. With the acceleration in urbanisation, industrialisation, ageing and lifestyle changes, the prevalence of cardiovascular disease (CVD) in China has dramatically increased and will remain on an upward trend in the next decades. ${ }^{1}$ According to a 2012 report on CVDs in China, an estimated 290 million individuals suffered from CVD and 3.5 million died of CVD every year, which accounted for $41 \%$ of all-cause deaths. ${ }^{2}$ CVD has become the top killer for the Chinese population. ${ }^{2}$ The increasing disease burden of CVD has become a major public health issue. The ongoing 
deterioration in cardiovascular risk factors (CRFs) appears to be the major cause of inducing the CVD epidemic in China. ${ }^{1}$

It is well known that cigarette smoking, overweight or obesity, hypertension, diabetes and dyslipidaemia are five major modifiable CRFs that can be altered or eliminated through proper management. ${ }^{3-11}$ A number of studies have indicated that the prevalence of CRFs has increased in recent decades in China. ${ }^{3-7} 9-11$ Furthermore, the clustering of these risk factors in the same individual will significantly increase risk of CVD events compared with a single risk factor. ${ }^{46-12}$ A complete assessment of the distribution and aggregate of well-established CRFs depicts the risk of developing CVD and is useful in formulating effective prevention strategies.

CVD is the leading cause of mortality in Nanjing, accounting for approximately $43 \%$ of total deaths in 2012. ${ }^{13}$ Although there have been similar studies previously in foreign countries, ${ }^{814-18}$ there has been no largescale study with an adequate sample size to evaluate the prevalence and clustering of CVD risk factors in Nanjing. In the present study, we performed a community-based survey to estimate the up-to-date prevalence and clustering of CVD risk factors, and investigate the association between relevant characteristics and CVD risk factor clustering among a large representative sample of the Nanjing adult population from eastern China.

\section{METHODS}

\section{Study population}

Data were drawn from Nanjing Chronic Disease and Risk Factor Surveillance (NCDRFS), which was a community-based cross-sectional study to obtain a regional representative sample of the general population aged $\geq 18$ years. The NCDRFS was conducted between June and November 2011 in Nanjing, the capital of Jiangsu Province in eastern China. Nanjing had a resident population of more than 8.1 million with eight urban districts and five rural counties in 2011. The sample size for the present study was calculated based on a prevalence (p) of diabetes of $5.8 \%$ among adults aged 18 years or older in Jiangsu Province, ${ }^{19}$ the design effect (deff) of 1.5 , an $\mathrm{u}$ value of 1.96 , a relative error $(\mathrm{r})$ of $5 \%$ and a non-response rate of $20 \%$, using the formula $n=\operatorname{deff} \times u^{2}(p(1-p) /$ $\left.(\mathrm{r} \times \mathrm{p})^{2}\right) \times(1+20 \%)$. We estimated a required sample size of approximately 45000 subjects.

Briefly, a multistage random sampling method was adopted. In the first stage, we covered all 13 administrative regions in Nanjing. In the second stage, 55 streets in urban districts and 45 towns in rural counties were all covered from each administrative region. In the third stage, three neighbourhood communities or administrative villages in each street or town were randomly selected with probability proportional to size. In the fourth stage, a resident group or villager group from each chosen neighbourhood community or administrative village was selected with a simple random sampling method. In the fifth stage, 150 households (a resident group containing about 250-500 households, and a villager group containing about 150-200 households) from each chosen resident group or villager group were randomly selected with a simple random sampling method. In the final stage, one person aged $\geq 18$ years, who was a local registered resident for more than 6 months was selected randomly from each chosen household using a Kish selection table. A total of 45000 individuals were selected, 41072 residents volunteered to participate in the survey, with an overall response rate of 91.3\% (41 072/45 000). We excluded 1232 residents due to missing complete information on CVD risk factors $(n=176)$ or having a self-reported history of CVD (coronary heart disease and stroke) $(n=1056)$. Finally, 39840 participants were included in the present analyses.

Written informed consent was obtained from each participant prior to the survey.

\section{Data collection and measurement}

At each surveillance point, trained staff collected data according to a standard protocol at local community health service centres/stations in the participants' registration address. The information included interview survey, physical measurements and laboratory examinations.

Standard questionnaire information included demographic characteristics (age, gender, address, and marriage), socioeconomic characteristics (education, occupation and annual family income), lifestyle risk factors (smoking, drinking status, physical activity (PA) and dietary habits) and their past medical history.

Physical measurements included weight, height and blood pressure (BP). Weight was measured with light clothing to the nearest $0.1 \mathrm{~kg}$ and height was measured without shoes to the nearest $0.1 \mathrm{~cm}$. Body mass index (BMI) was calculated as weight in kilograms divided by the square of the height in metres. Participants were advised to avoid smoking cigarettes, drinking alcohol, taking tea or coffee or engaging in PA for at least 30 min before BP measurements. BP was measured three times by trained professionals after at least $5 \mathrm{~min}$ of rest in the seated position, using an electronic sphygmomanometer (OMRON HEM-7200, Japan). Three recordings were made at 2 min intervals. The average of the second and third BP measurements was used for the analysis.

Blood samples were collected from all subjects in the morning after overnight fasting at least 10 hours. Laboratory examinations included fasting blood glucose and four items of blood lipids. The laboratory completed the blood examinations within 8 hours of receiving the samples. Fasting plasma glucose (FPG) was measured enzymatically using a glucose oxidase method. Serum lipids, including total cholesterol (TC), triglycerides (TG), low-density lipoprotein cholesterol (LDL-C) and high-density lipoprotein cholesterol (HDL-C) were measured using an autoanalyser (Abbott Laboratories, Illinois, USA). 


\section{Assessment criteria}

\section{Cardiovascular risk factors}

Five major CVD risk factors were defined based on current national guidelines. Hypertension was defined as self-reported current treatment with antihypertensive medication in the past 2 weeks, and/or an average systolic BP (SBP) $\geq 140 \mathrm{~mm} \mathrm{Hg}$ and/or an average diastolic BP (DBP) $\geq 90 \mathrm{~mm}$ Hg. ${ }^{20}$ Diabetes was defined as self-reported current treatment with antidiabetes medication (insulin or oral hypoglycaemic agents) and/or FPG $\geq 7.0 \mathrm{mmol} / \mathrm{L}{ }^{21}$ Dyslipidaemia was defined as self-reported current treatment with antilipaemic medication or having at least one of the following: $\mathrm{TG} \geq 2.26 \mathrm{mmol} / \mathrm{L}, \mathrm{TC} \geq 6.22 \mathrm{mmol} / \mathrm{L}, \mathrm{LDL}-\mathrm{C} \geq 4.14 \mathrm{mmol} / \mathrm{L}$ and HDL-C $<1.04 \mathrm{mmol} / \mathrm{L}^{22}$ Overweight or obesity were defined as $\mathrm{BMI} \geq 24.0 \mathrm{~kg} / \mathrm{m}^{2}{ }^{23}$ Current smoking was defined as having smoked at least one cigarette daily continuously or at least 18 packs in total each year. ${ }^{24}$ Both ex-smokers (those who smoked previously but quit subsequently) and those who never smoked cigarettes were categorised as non-smokers in our study. CVD risk factor clustering was defined as having at least two risk factors in one individual. ${ }^{412}$

\section{Covariates}

Occupation, income and education were taken into the assessment of socioeconomic status (SES). Occupation was recorded into four groups: manual labourers (workers, farmers, fishermen and herdsmen), service staff (service personnel and housekeepers), mental labourers (professionals, office clerk and other technical staff) and others (unemployed and retired people).${ }^{25}$ The total annual family income was divided into tertiles: lower, middle and higher. ${ }^{26}$ Education was grouped into three categories: primary school and lower, junior or senior high school, and college and higher.

The validated Chinese short-version of the International Physical Activity Questionnaire was used to measure self-reported PA in the past week. ${ }^{27}$ Total PA time was calculated as the sum of the time spent in moderate-intensity PA plus double the time spent in vigorous-intensity PA according to PA guidelines for Americans. ${ }^{28}$ Participants who engaged in $\geq 150 \mathrm{~min} /$ week of total PA time were classified as having sufficient PA, and those who engaged in $<150 \mathrm{~min} /$ week of total PA time were classified as having insufficient PA. ${ }^{28}$

A semiquantitative Food Frequency Questionnaire (FFQ) was used to assess dietary intake during the previous year. The reliability and validity of the FFQ have been examined elsewhere. ${ }^{29}$ Subjects were defined as having unhealthy dietary habits if they had at least three of the following per day: excessive intake of salt ( $>6 \mathrm{~g} /$ day), excessive intake of red meat $(>100 \mathrm{~g} /$ day $)$, insufficient intake of vegetables and fruits $(<400 \mathrm{~g} /$ day $)$, insufficient intake of soya-based foods $(<25 \mathrm{~g} /$ day $)$ and insufficient intake of dairy products $(<300 \mathrm{~g} /$ day $) .{ }^{30-32}$

\section{Quality control}

In order to ensure the reliability of the investigation data, a vigorous quality control was implemented by
Nanjing Municipal Center for Disease Control and Prevention (Nanjing CDC). The quality control was conducted throughout the survey period, including design and revision of the preliminary plan, unifying investigation tools, preparing standard training materials and technical requirements, conducting field supervision and technical guidance, extracting 10\% questionnaire for verification and 5\% respondents to review their physical measurements at each surveillance points, and data cleaning and analysis. The coincidence rate was over $95 \%$. The feedback and correction was made timely when the problems were found. All study investigators completed a uniform training programme and passed the examination at the end of the training. A manual of procedures was distributed by Nanjing CDC, and detailed instructions for administration of the questionnaires, anthropometric measurements and biological specimen collection were provided. All data were entered twice and validated.

\section{Statistical analysis}

The data were presented as means (SD) for continuous variables, and as percentages $(95 \% \mathrm{CI})$ for categorical variables. Differences in continuous variables were analysed using $t$ test, and prevalence values for categorical variables were compared using $\chi^{2}$ test. The study data were weighted by 12 age groups (5-year intervals) and two gender groups (men or women) according to the Nanjing sixth national population census, which allowed for calculation of regional representative estimates. ${ }^{341233}$ The weighted prevalence of each CVD risk factor and presence of cardiovascular risk factors (CFRs) $(0,1$ or $\geq 2)$ were described in the overall population and stratified by gender. The association of relevant characteristics with CVD risk factor clustering was performed by multinomial logistic regression models. The adjusted ORs and 95\% CIs of having one, two or more CVD risk factors versus no risk factor were analysed using multivariate logistic regression models. Variables that were statistically significant at $\mathrm{p}<0.05$ in the univariate analysis were entered into the multivariate logistic regression analyses.

All statistical analyses were performed using SPSS software V.20.0 (IBM, Armonk, New York, USA). A two-tailed $\mathrm{p}<0.05$ was considered statistically significant.

\section{Patient involvement}

Patients were not involved in setting the research question, the outcome measures, the design or the implementation of the study. No patients were asked to advise on interpretation or writing up of results. No patients were advised on dissemination of the present study and its main results.

\section{RESULTS}

As shown in table 1 , in a total of 39840 participants (18-86 years, mean age $47.9 \pm 16.2$ years), 17964 (45.1\%) were men and 21876 (54.9\%) were women. More than four-fifths $(84.6 \%)$ of the residents were married, nearly half $(47.9 \%)$ were manual labourers, $50.4 \%$ had 
Table 1 Descriptive characteristics of participants in Nanjing, China

\begin{tabular}{|c|c|c|c|}
\hline Characteristic & Men (n=17 964) & Women $(n=21876)$ & Total $(n=39840)$ \\
\hline Number, n (\%) & $17964(45.1)$ & $21876(54.9)$ & $39840(100.0)$ \\
\hline Age, years, mean (SD) & $48.0 \pm 16.4$ & $47.8 \pm 16.2$ & $47.9 \pm 16.2$ \\
\hline \multicolumn{4}{|l|}{ Marriage, $\mathrm{n}(\%)^{*}$} \\
\hline Single & $2167(12.1)$ & $1877(8.6)$ & $4044(10.2)$ \\
\hline Married or living with a partner & $15174(84.5)$ & $18537(84.7)$ & $33711(84.6)$ \\
\hline Separated, divorced or widowed & $623(3.5)$ & $1462(6.7)$ & $2085(5.2)$ \\
\hline \multicolumn{4}{|l|}{ Education, $\mathrm{n}(\%)^{*}$} \\
\hline Primary school and lower & $3865(21.5)$ & $8114(37.1)$ & $11979(30.1)$ \\
\hline Junior or senior high school & $10045(55.9)$ & $10059(46.0)$ & $20104(50.4)$ \\
\hline College and higher & $4054(22.6)$ & $3703(16.9)$ & 7757 (19.5) \\
\hline \multicolumn{4}{|l|}{ Occupation, $\mathrm{n}(\%)^{*}$} \\
\hline Manual labourers & $9090(50.6)$ & $10002(45.7)$ & $19092(47.9)$ \\
\hline Service staff & $1002(5.6)$ & $1474(6.7)$ & $2476(6.2)$ \\
\hline Mental labourers & 3501 (19.5) & $2876(13.1)$ & $6377(16.0)$ \\
\hline Unemployed and retired people & $4371(24.3)$ & $7524(34.4)$ & $11895(29.9)$ \\
\hline \multicolumn{4}{|l|}{ Annual family income, $\mathrm{n}(\%)^{\star}$} \\
\hline Lower & $4420(24.6)$ & $5978(27.4)$ & $10398(26.1)$ \\
\hline Middle & $7580(42.2)$ & $9352(42.7)$ & $16932(42.5)$ \\
\hline Higher & $5964(33.2)$ & $6546(29.9)$ & $12510(31.4)$ \\
\hline \multicolumn{4}{|l|}{ Residence, $\mathrm{n}(\%)^{*}$} \\
\hline Urban & $7845(43.7)$ & $8456(38.7)$ & $16301(40.9)$ \\
\hline Rural & $10119(56.3)$ & $13420(61.3)$ & $23539(59.1)$ \\
\hline \multicolumn{4}{|l|}{ Physical activity, $\mathrm{n}(\%)^{*}$} \\
\hline Insufficient & $12247(68.2)$ & $13128(60.0)$ & $25375(63.7)$ \\
\hline Sufficient & 5717 (31.8) & $8748(40.0)$ & $14465(36.3)$ \\
\hline \multicolumn{4}{|l|}{ Dietary habits, $\mathrm{n}(\%)^{*}$} \\
\hline Unhealthy & $13082(72.8)$ & $16283(74.4)$ & $29365(73.7)$ \\
\hline Healthy & $4882(27.2)$ & $5593(25.6)$ & $10475(26.3)$ \\
\hline $\mathrm{BMI}, \mathrm{kg} / \mathrm{m}^{2}$, mean $(\mathrm{SD})^{*}$ & $23.6 \pm 3.0$ & $23.2 \pm 3.3$ & $23.4 \pm 3.2$ \\
\hline SBP, mm Hg, mean (SD) ${ }^{*}$ & $125.1 \pm 14.7$ & $121.8 \pm 16.8$ & $123.3 \pm 16.0$ \\
\hline DBP, mm Hg, mean (SD) & $80.1 \pm 9.3$ & $77.6 \pm 9.7$ & $78.7 \pm 9.6$ \\
\hline FPG, mmol/L, mean (SD) & $5.3 \pm 1.3$ & $5.3 \pm 1.3$ & $5.3 \pm 1.3$ \\
\hline TC, mmol/L, mean (SD)† & $4.7 \pm 1.7$ & $4.5 \pm 1.5$ & $4.6 \pm 1.3$ \\
\hline $\mathrm{TG}, \mathrm{mmol} / \mathrm{L}$, mean $(\mathrm{SD})^{*}$ & $1.6 \pm 0.6$ & $1.4 \pm 0.5$ & $1.5 \pm 0.9$ \\
\hline
\end{tabular}

${ }^{*} p<0.01$ when comparing men with women.

$\mathrm{tp}<0.05$ when comparing men with women.

BMI, body mass index; DBP, diastolic blood pressure; FPG, fasting plasma glucose; SBP, systolic blood pressure; TC, total cholesterol; TG, triglycerides.

an education level of junior or senior high school, and $42.5 \%$ had a middle level of annual family income. Nearly $60 \%(59.1 \%)$ of adults were from rural areas. The proportions of participants with sufficient PA and healthy dietary habits were $36.3 \%$ and $26.3 \%$, respectively. Compared with women, men had a higher level of BMI, SBP, DBP, TC and TG (all $\mathrm{p}<0.01)$.

The weighed prevalence of hypertension, diabetes, dyslipidaemia, overweight or obesity, and current smoking was $23.9 \%, 6.2 \%, 28.9 \%, 35.6 \%$ and $24.5 \%$, respectively. The weighted prevalence of hypertension, dyslipidaemia, overweight or obesity, and current smoking was higher in men than in women (all $\mathrm{p}<0.001$ ), except for diabetes. The weighed prevalence of these risk factors increased significantly with age for the general population regardless of gender (all $\mathrm{p}<0.001)$. The proportion of CRFs tended to be higher among participants who lost a life partner (all $\mathrm{p}<0.001)$. The prevalence of CRFs decreased 
Open access

Table 2 The weighted prevalence $(95 \% \mathrm{Cl})$ of five major cardiovascular disease (CVD) risk factors by relevant characteristics

\begin{tabular}{|c|c|c|c|c|c|}
\hline Category & Hypertension & Diabetes & Dyslipidaemia & $\begin{array}{l}\text { Overweight or } \\
\text { obesity }\end{array}$ & Smoking \\
\hline Total & 23.9 (23.5 to 24.3$)$ & $6.2(6.0$ to 6.4$)$ & 28.9 (28.5 to 29.3$)$ & 35.6 (35.1 to 36.1$)$ & 24.5 (24.1 to 24.9$)$ \\
\hline \multicolumn{6}{|l|}{ Gender } \\
\hline Men & 25.4 (24.8 to 26.0$)$ & 6.2 (5.8 to 6.6$)$ & 31.5 (30.8 to 32.2$)$ & 39.6 (38.9 to 40.3 ) & 46.1 (45.4 to 46.8$)$ \\
\hline Women & $22.2(21.6$ to 22.8$)$ & 6.1 (5.8 to 6.4$)$ & 26.2 (25.6 to 26.8$)$ & 31.4 (30.8 to 32.0$)$ & 1.5 (1.3 to 1.7$)$ \\
\hline$P$ value & $<0.001$ & $>0.05$ & $<0.001$ & $<0.001$ & $<0.001$ \\
\hline $18-34$ & 5.3 (4.9 to 5.7$)$ & $1.6(1.4$ to 1.8$)$ & $19.0(18.3$ to 19.7$)$ & 21.8 (21.0 to 22.6$)$ & 16.6 (15.9 to 17.3$)$ \\
\hline $35-59$ & 27.7 (27.1 to 28.3$)$ & $6.9(6.5$ to 7.3$)$ & 34.4 (33.7 to 35.1$)$ & 44.5 (43.8 to 45.2$)$ & 31.8 (31.1 to 32.5$)$ \\
\hline$\geq 60$ & 56.8 (55.9 to 57.7$)$ & 14.8 (14.1 to 15.5$)$ & 38.0 (37.1 to 38.9$)$ & 45.6 (44.7 to 46.5$)$ & 24.3 (23.5 to 25.1$)$ \\
\hline$P$ value & $<0.001$ & $<0.001$ & $<0.001$ & $<0.001$ & $<0.001$ \\
\hline $\begin{array}{l}\text { Separated to divorced, or } \\
\text { widowed }\end{array}$ & $51.0(48.9$ to 53.1$)$ & $12.2(10.8$ to 13.6$)$ & 32.7 (30.7 to 34.7$)$ & 42.0 (39.9 to 44.1$)$ & 18.9 (17.2 to 20.6$)$ \\
\hline$P$ value & $<0.001$ & $<0.001$ & $<0.001$ & $<0.001$ & $<0.001$ \\
\hline \multicolumn{6}{|l|}{ Education } \\
\hline Primary school and lower & 42.8 (41.9 to 43.7$)$ & 10.9 (10.3 to 11.5$)$ & 33.9 (33.1 to 34.7$)$ & 45.6 (44.7 to 46.5$)$ & 20.2 (19.5 to 20.9$)$ \\
\hline Junior or senior high school & $22.8(22.2$ to 23.4$)$ & $5.8(5.5$ to 6.1$)$ & 30.1 (29.5 to 30.7$)$ & 37.2 (36.5 to 37.9 ) & 29.8 (29.2 to 30.4$)$ \\
\hline College and higher & 10.2 (9.5 to 10.9$)$ & 2.9 (2.5 to 3.3$)$ & 22.5 (21.6 to 23.4$)$ & 24.4 (23.4 to 25.4$)$ & 17.8 (16.9 to 18.7$)$ \\
\hline$P$ value & $<0.001$ & $<0.001$ & $<0.001$ & $<0.001$ & $<0.001$ \\
\hline \multicolumn{6}{|l|}{ Occupation } \\
\hline \multicolumn{6}{|l|}{ Annual family income } \\
\hline Lower & 35.1 (34.2 to 36.0$)$ & $8.9(8.4$ to 9.4$)$ & 34.9 (34.0 to 35.8$)$ & 42.5 (41.5 to 43.5$)$ & 29.1 (28.2 to 30.0$)$ \\
\hline Middle & 21.7 (21.1 to 22.3$)$ & 5.9 (5.5 to 6.3$)$ & 31.3 (30.6 to 32.0$)$ & 37.0 (36.3 to 37.7$)$ & 25.9 (25.2 to 26.6$)$ \\
\hline Higher & $17.4(16.7$ to 18.1$)$ & 4.8 (4.4 to 5.2$)$ & 26.4 (25.6 to 27.2$)$ & 33.4 (32.6 to 34.2 ) & 22.2 (21.5 to 22.9$)$ \\
\hline$P$ value & $<0.001$ & $<0.001$ & $<0.001$ & $<0.001$ & $<0.001$ \\
\hline \multicolumn{6}{|l|}{ Residence } \\
\hline Urban & 23.6 (22.9 to 24.3$)$ & 5.9 (5.5 to 6.3$)$ & 28.4 (27.7 to 29.1$)$ & 35.4 (34.7 to 36.1$)$ & 24.7 (24.0 to 25.4$)$ \\
\hline Rural & 24.0 (23.5 to 24.5$)$ & $6.5(6.2$ to 6.8$)$ & 29.3 (28.7 to 29.9 ) & 35.8 (35.2 to 36.4 ) & 24.3 (23.8 to 24.8$)$ \\
\hline$P$ value & $<0.05$ & $<0.01$ & $<0.01$ & $>0.05$ & $>0.05$ \\
\hline
\end{tabular}

with the increasing level of education and family income (all $\mathrm{p}<0.001$ ). The prevalence of hypertension, diabetes and dyslipidaemia was the highest in unemployed and retired people (all $\mathrm{p}<0.001)$. Moreover, the prevalence of hypertension, diabetes and dyslipidaemia was greater in rural areas than in urban areas (tables 2 and3).

Overall, 34.7, 30.1\%, 20.8\%, $10.6 \%, 3.3 \%$ and $0.5 \%$ of Nanjing adults had zero, one, two, three, four and five risk factors, respectively. In total $65.3 \%$ and $35.2 \%$ of the population had at least one or at least two CVD risk factors. There were $23.4 \%$ and $46.8 \%$ of men and women having no risk factor, respectively. In contrast, $31.4 \%$ and $45.2 \%$ of men, and $28.7 \%$ and $24.6 \%$ of women had one and at least two of these risk factors, respectively. The weighted prevalence of at least two risk factors was higher in men than in women $(p<0.001)$. The prevalence of at least two CVD risk factors increased with age for the overall population of both genders (all p <0.001). Regardless of men and women, there was a decreasing trend towards CVD risk 


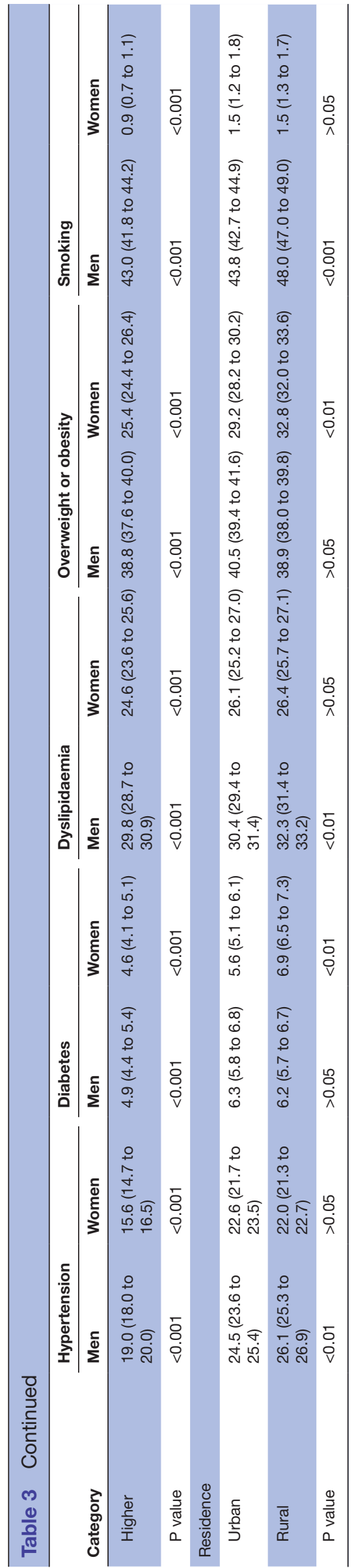

factor clustering with increasing levels of education and yearly family income (all $\mathrm{p}<0.001$ ). In addition, the clustering of CVD risk factors was higher among unemployed and retired people, and those with insufficient PA or unhealthy diets compared with their counterparts, respectively (all $\mathrm{p}<0.001$ ) (tables 4 andTable 5 ).

The combinations of dyslipidaemia/overweight or obesity, hypertension/overweight or obesity, and current smoking/overweight or obesity were the three most common of all two risk factors clustering; the proportions were $22.4 \%, 20.5 \%$ and $18.2 \%$, respectively. Moreover, the clustering of hypertension/dyslipidaemia/overweight or obesity, and the clustering of hypertension/dyslipidaemia/overweight or obesity/ current smoking were the most frequent among combinations of three or four risk factors; their proportions were $32.6 \%$ and $58.0 \%$, respectively.

As shown in table 6 , the multinomial logistic regression analysis showed that men, the elderly, rural residents, participants who had lower levels of SES or those who had lost a life partner, and those with insufficient PA or unhealthy diets were more likely to have one or at least two CVD risk factors compared with women, the young, urban residents, participants who had higher levels of SES or were single, and those with sufficient PA or healthy diets, respectively.

\section{DISCUSSION}

With economic development and urbanisation changes in lifestyle behaviours, China has experienced a rapid increase in the prevalence of CVD and its related risk factors. To our knowledge, the present study is the first study to estimate the up-to-date prevalence and clustering of major CVD risk factors among a large representative sample of the Nanjing adult population from eastern China.

In this community-based cross-sectional study, we found that the prevalence of overweight or obesity, dyslipidaemia, and smoking were ranked as the top three CVD risk factors in the general population. The most prominent risk factor among men and women was smoking and overweight or obesity, respectively. The prevalence of overweight or obesity in our study was much lower than that observed in the nationwide population ${ }^{34}$ and some other regional studies, ${ }^{45}$ but our findings were close to a recent Tibetan population study. ${ }^{3}$ Moreover, the prevalence of dyslipidaemia was significantly lower than the national level in a recent cross-sectional study. ${ }^{36}$ The present study showed that the prevalence of smoking was lower than that in the China Global Adults Tobacco Survey of 2010. ${ }^{37}$ Additionally, the prevalence of these CRFs among Nanjing adults was similar to that in other developed Asian countries, ${ }^{17} 18$ but was lower than in developing countries. ${ }^{1415}$

Emerging evidence revealed that the synergistic effect of CVD risk factor clustering was associated with a higher risk of developing preclinical CVD and CVD events. ${ }^{38}$ Previous studies confirmed that the clustering of CRFs had more harmful cardiovascular effects than a single 
Table 4 The weighted prevalence $(95 \% \mathrm{Cl})$ with different numbers of cardiovascular disease (CVD) risk factors

\begin{tabular}{|c|c|c|c|c|}
\hline Category & None (0) & Single (1) & Clustering ( $\geq 2)$ & $P$ value \\
\hline Total & 34.7 (34.2 to 35.2$)$ & 30.1 (29.6 to 30.6 ) & $35.2(34.7$ to 35.7$)$ & \\
\hline Gender & & & & $<0.001$ \\
\hline Men & 23.4 (22.8 to 24.0$)$ & 31.4 (30.7 to 32.1 ) & $45.2(44.5$ to 45.9$)$ & \\
\hline Women & 46.8 (46.1 to 47.5$)$ & 28.7 (28.1 to 29.3 ) & 24.6 (24.0 to 25.2$)$ & \\
\hline Age group, years & & & & $<0.001$ \\
\hline $18-34$ & 55.2 (54.2 to 56.2$)$ & 29.8 (28.9 to 30.7$)$ & 15.0 (14.3 to 15.7$)$ & \\
\hline $35-59$ & 24.4 (23.8 to 25.0$)$ & 31.1 (30.5 to 31.7$)$ & 44.5 (43.8 to 45.2$)$ & \\
\hline$\geq 60$ & $13.3(12.7$ to 13.9$)$ & 28.3 (27.4 to 29.2 ) & $58.4(57.5$ to 59.3$)$ & \\
\hline Marriage & & & & $<0.001$ \\
\hline Single & 59.8 (58.3 to 61.3$)$ & 27.4 (26.0 to 28.8$)$ & 12.8 (11.8 to 13.8$)$ & \\
\hline Married or living with a partner & 29.0 (28.5 to 29.5$)$ & 30.8 (30.3 to 31.3 ) & 40.2 (39.7 to 40.7$)$ & \\
\hline Separated to divorced, or widowed & 19.0 (17.3 to 20.7$)$ & 30.2 (28.2 to 32.2 ) & 50.8 (48.7 to 52.9$)$ & \\
\hline Education & & & & $<0.001$ \\
\hline Primary school and lower & 20.8 (20.1 to 21.5$)$ & 30.9 (30.1 to 31.7$)$ & 48.4 (47.5 to 49.3$)$ & \\
\hline Junior or senior high school & 32.4 (31.8 to 33.0$)$ & 30.3 (29.7 to 30.9$)$ & 37.3 (36.6 to 38.0$)$ & \\
\hline College and higher & 50.8 (49.7 to 51.9$)$ & 29.0 (28.0 to 30.0$)$ & 20.2 (19.3 to 21.1$)$ & \\
\hline Occupation & & & & $<0.001$ \\
\hline Manual labourers & 31.0 (30.3 to 31.7$)$ & 30.6 (29.9 to 31.3$)$ & 38.4 (37.7 to 39.1$)$ & \\
\hline Service staff & 41.4 (39.5 to 43.3 ) & 31.8 (30.0 to 33.6 ) & 26.8 (25.1 to 28.5$)$ & \\
\hline Mental labourers & 42.4 (41.2 to 43.6$)$ & 31.0 (29.9 to 32.1$)$ & 26.6 (25.5 to 27.7$)$ & \\
\hline Unemployed and retired people & 34.0 (33.1 to 34.9$)$ & 28.0 (27.2 to 28.8 ) & 38.0 (37.1 to 38.9$)$ & \\
\hline Family income yearly & & & & $<0.001$ \\
\hline Lower & 23.1 (22.3 to 23.9 ) & 28.0 (27.1 to 28.9 ) & 46.8 (45.8 to 47.8$)$ & \\
\hline Middle & 33.8 (33.1 to 34.5$)$ & 31.2 (30.5 to 31.9$)$ & $35.0(34.3$ to 35.7$)$ & \\
\hline Higher & 36.6 (35.8 to 37.4$)$ & 30.8 (30.0 to 31.6$)$ & 32.6 (31.8 to 33.4$)$ & \\
\hline Residence & & & & $>0.05$ \\
\hline Urban & 34.8 (34.1 to 35.5$)$ & 30.3 (29.6 to 31.0 ) & 35.0 (34.3 to 35.7$)$ & \\
\hline Rural & 34.7 (34.1 to 35.3$)$ & 29.9 (29.3 to 30.5$)$ & 35.4 (34.8 to 36.0$)$ & \\
\hline Physical activity & & & & $<0.05$ \\
\hline Insufficient & 34.0 (33.4 to 34.6$)$ & 30.5 (30.0 to 31.1) & 35.5 (34.9 to 36.1$)$ & \\
\hline Sufficient & 36.2 (35.4 to 37.0$)$ & 29.2 (28.5 to 29.9$)$ & 34.6 (33.8 to 35.4$)$ & \\
\hline Dietary habits & & & & $<0.05$ \\
\hline Unhealthy & 34.7 (34.2 to 35.2 ) & 29.6 (29.1 to 30.1 ) & 35.7 (35.2 to 36.2$)$ & \\
\hline Healthy & 34.9 (34.0 to 35.8$)$ & 31.4 (30.5 to 32.3$)$ & 33.6 (32.7 to 34.5$)$ & \\
\hline
\end{tabular}

risk factor. ${ }^{4}{ }^{9-12}$ Risk for CVD and all-cause mortality increased substantially with each additional risk factor. Data from the First National Health and Nutrition Examination Surveys Epidemiologic Follow-up Study showed that more than $50 \%$ of the incidence of coronary heart disease, stroke and all-cause mortality was attributable to having one risk factor, while the attributable risk was more than $70 \%$ for participants with three risk factors. ${ }^{40}$ Therefore, assessment of CVD risk factor clustering is crucial to identify a high-risk population.

In the present study, $34.7 \%, 30.1 \%$ and $35.2 \%$ of participants had zero, one, and two or more major
CRFs, respectively, among residents aged $\geq 18$ years. A higher level of clustering of the five CVD risk factors was found in the general Chinese population over the last decade. In a nationally representative sample of 46 239 adults aged $\geq 20$ years from the 2007-2008 China National Diabetes and Metabolic Disorders Study, the proportions of respondents with zero, one and at least two CVD risk factors were $13.5 \%, 31.2 \%$ and $55.3 \%$, respectively. ${ }^{41}$ In another national study of 23010 Chinese adults $\geq 18$ years from the 2007-2011 Chinese Physiological Constant and Health Condition, 29.7\%, $30.0 \%$ and $40.3 \%$ of participants had zero, one and at 


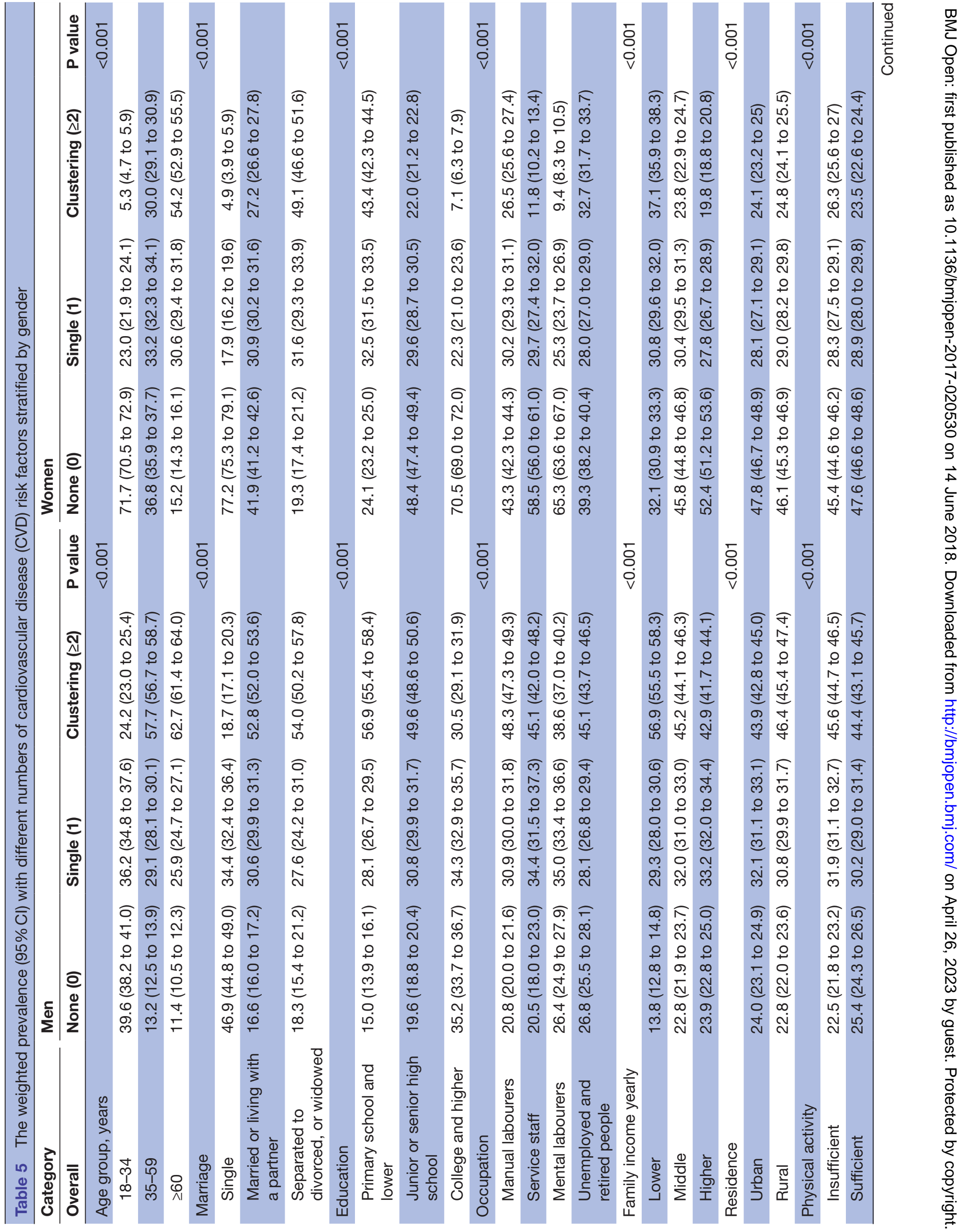


least two CRFs, respectively. ${ }^{6}$ Compared with these two national studies mentioned above, ${ }^{641}$ Nanjing residents had a lower proportion of two or more CVD risk factors and a higher proportion of no risk factor.

Our results were similar to other related studies in China. ${ }^{12} 42$ Among 46683 Chinese from the 2009-2010 China National Survey of chronic kidney disease, the prevalence of having at least two risk factors was $36.2 \%{ }^{12}$ Overall, $30.9 \%, 36.4 \%$ and $32.7 \%$ of participants had zero, one and at least two CRFs, respectively, in 37141 adults $\geq 18$ years in a healthy screening population of Changchun in the Jilin Province of North-East China. ${ }^{42}$ Unfortunately, since these two studies did not include current smoking as a CVD risk factor, the clustering of CVD risk factors may underestimate the actual value. We also found that the clustering of dyslipidaemia/overweightor obesity, hypertension/dyslipidaemia/overweight or obesity, and hypertension/dyslipidaemia/overweight or obesity/current smoking were the most common among combinations of two, three and four risk factors, respectively.

Clustering of CVD risk factors has also been observed in other Asian countries that have experienced rapid socioeconomic growth like China. ${ }^{15-18}$ Clustering of three or more CVD risk factors was presented in $22.7 \%$ of the men and $21.7 \%$ of the women in South Korea. ${ }^{17}$ In Malaysia, $33 \%$ of the national population had two or more risk factors. ${ }^{18}$ The proportions of adults with two, three and at least four risk factors were $23.1 \%, 15.5 \%$ and $8.4 \%$, respectively, in South-Western Nigeria. ${ }^{16}$ A total of $78.6 \%$ subjects had two or more risk factors in different parts of India. ${ }^{15}$

Our study showed that men and the elderly were more likely to have two or more CVD risk factors compared with women and the young, in accordance with other previous studies. ${ }^{3} 671011$ The gender disparities may be due to the fact that Chinese men assume more responsibility in society, tend to smoke more cigarettes and be more susceptible to psychological stress than women. In contrast, Chinese women tend to have more awareness of body weight especially in their young and middle years, which could translate into favourable cardiovascular risk profiles. However, the sex discrepancy in CVD risk factor clustering does not exist in other economically developed countries. In both South Korea and Malaysia, ${ }^{17} 18$ the prevalence of obesity in women was significantly higher than in men, and clustering of CRFs was more common in women. The result that the clustering of CVD risk factors increased progressively with age may attribute to the increasing prevalence of each risk factor with age. Our data showed that the clustering of CVD risk factors was more common in rural areas than in urban areas, inconsistent with other previous studies. ${ }^{59}$ Urban-rural differences may be explained by the rapid urbanisation of rural areas, and the improvement of the healthcare system and health conditions in urban areas in China.

Our study demonstrated that the level of SES was negatively associated with CVD risk factor clustering. As the level of SES increased, the number of CVD risk factors 
Table 6 The multinomial logistic analysis of the cardiovascular disease (CVD) risk factor clustering

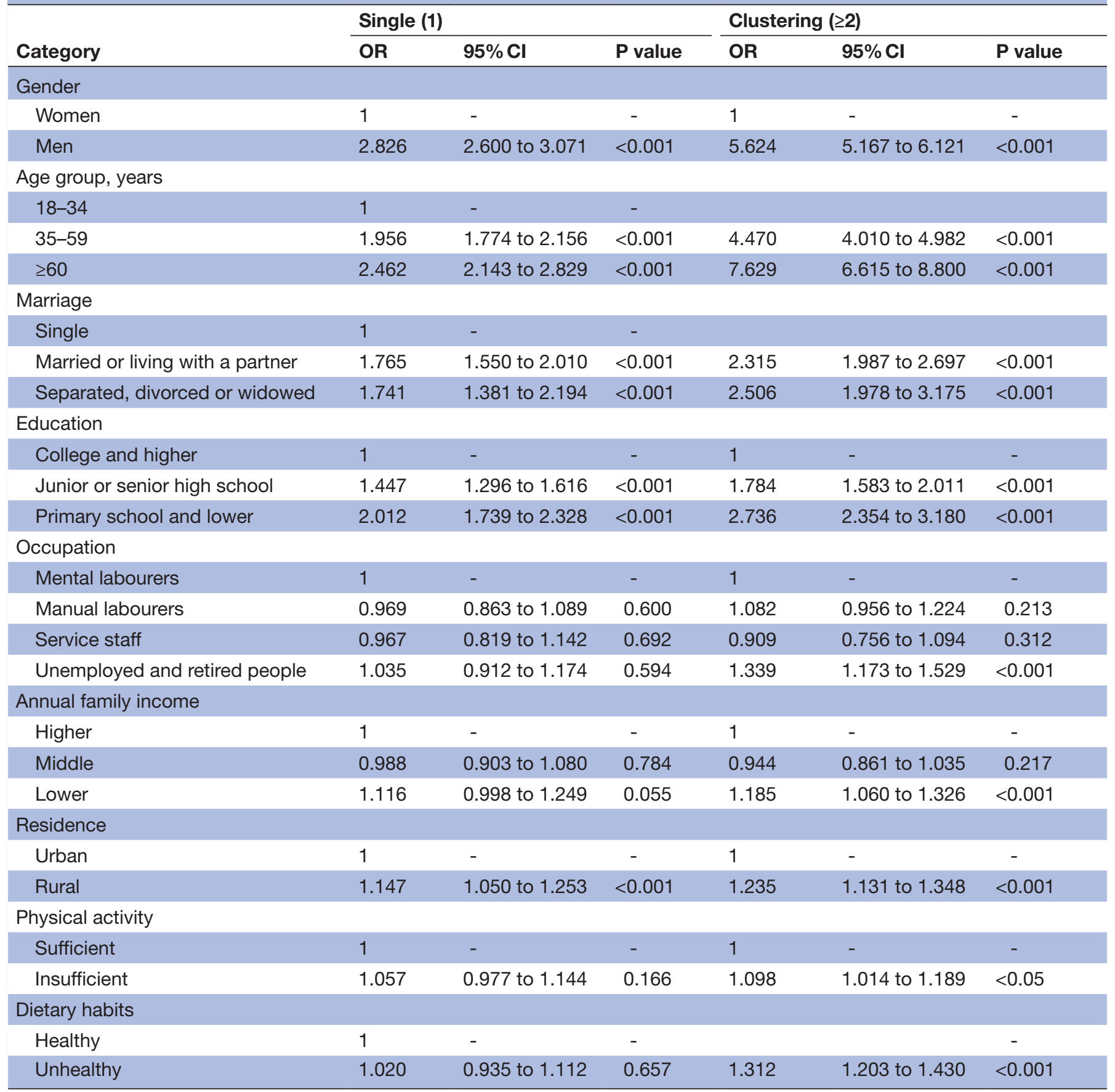

A multivariate logistic regression model was used to estimate ORs with 95\% Cls, and all other factors were adjusted when ORs with $95 \% \mathrm{Cls}$ of each variable were estimated.

decreased, which was consistent with other reports. ${ }^{3} 4812$ Socioeconomic inequalities may have unequal access to the knowledge of chronic disease control and prevention, healthcare services and medical resources. Socioeconomic disparities may eventually lead to health behaviours inequality with various SES levels.

The present study also showed that insufficient PA and unhealthy diets were positively associated with the clustering of CVD risk factors. Unhealthy lifestyle behaviours were still prevalent in Nanjing, in line with other domestic studies. ${ }^{43}{ }^{44}$ Unhealthy lifestyle behaviours may play an important role in the process of atherosclerosis by leading to physiological changes. ${ }^{4546}$ Lifestyle modification was the most important strategy in preventing CVD risk factors under the supervision of the government. In 2012, the blueprint for non-communicable diseases (NCDs) prevention and control in China (2012-2015) issued by 15 multispectral ministries including the Ministry of National Health and Family Planning was implemented, which promoted a nationwide healthy lifestyle initiative. A new operating paradigm for NCD prevention and control has been formed, featuring the government's 
leading role, multisectoral coordination, social mobilisation and extensive participation.

In the Health Nanjing 2030 programme, the Nanjing Municipal Health and Family Planning Commission has stated strong support for four main NCDs (CVD, diabetes, cancer and chronic respiratory disease) prevention and control, and is planning a corresponding regional health policy. First, only 18 cities have legislated to ban smoking in public areas at present, not including Nanjing city. The implementation of the smoking ban in public places will be promoted, and complete smoke-free indoor public places is gradually being accomplished in Nanjing. By 2020, all of the schools, party and government organisations, enterprises and institutions, medical and health agencies are required to be smoke-free. The overall smoking rate over 15 years of age will drop to $20.0 \%$ in 2030 . Second, the physical fitness awareness of all residents in Nanjing will be further enhanced; the physical quality and health level of people will be continuously improved by 2030. All communities or villages will provide practical basic public sports and fitness facilities, and an urbanrural ' 15 min fitness circle' shall be completely formed. Meanwhile, it is required to make efforts to provide free or low-cost sports fields and facilities, and public sports facilities and sports grounds of schools meeting conditions shall be $100 \%$ open to the public. Third, healthy nutrient products should be available to the public, especially green, organic, pollution-free and proteinrich foods. Nutrition and health knowledge should be spread and popularised. The use of salt, oil and sugar in processed foods should be controlled and reduced. The daily intake of salt in 2030 will be reduced relatively by $20 \%$ from the 2011 level in Nanjing. ${ }^{47}$ Final, more behaviour modification efforts are required to achieve CVD risk factor reduction. The effectively primary, secondary and tertiary prevention of CVD risk factors can substantially decrease the risk of developing CVD.

An advantage of the present study was that the results were based on a community-based study with a large representative sample of residents in Nanjing, which ensured sufficient power for calculation of regional estimates. Additionally, the high-quality study design and implementation with a high response rate, application of standard protocols and instruments, data collection by trained interviewers, and vigorous quality control throughout the survey period, in all the above ensured the validity of our self-reported data.

Our study also had several limitations. First, the cross-sectional design cannot determine the causality or temporal relationship between the clustering of CVD risk factors and CVD. However, previous studies demonstrated that these risk factors were more likely to develop CVD events in Chinese adults. ${ }^{48}$ Second, the oral glucose tolerance test was not performed in the present study, which could underestimate the prevalence of diabetes. Third, the information on current smoking status was based on self-report, which could be subject to reporting bias. Moreover, those who smoked one cigarette per day irregularly or less than 18 packs in total each year were not included in current smokers. Therefore, the prevalence of current smoking may be under-reported. Final, the mean of the second and third BP measurements in single-visit BP measurements was calculated for analyses, which could overestimate the prevalence of hypertension.

In summary, this cross-sectional study provides a regional prevalence and clustering of CVD risk factors in Nanjing, and fills the information gap in this region. Our analyses indicate that men, elderly people, rural residents and participants with lower levels of SES or unhealthy lifestyle behaviours are susceptible to CVD modifiable risk factor clustering, known as high-risk groups. It is important to focus on the prevention of CVD risk factors among the overall population and especially the vulnerable population in China. Consequently, the Nanjing government has developed effective public health policies at the regional level, such as avoidance or cessation of smoking in public areas, popularising scientific knowledge, enhancing the public's health awareness, organising health promotion programmes, producing healthy nutrient foods, providing free or low-cost public sports facilities and fitness facilities, strengthening major chronic diseases prevention and control, and improving the medical service system and basic public health service, to reduce the prevalence and clustering of CVD risk factors.

Acknowledgements The authors thank all the dedicated fieldworkers who took part in the surveys and all participants who facilitated the survey implementation at each community.

Contributors $\mathrm{XH}, \mathrm{QY}, \mathrm{JH}, \mathrm{FX}$ contributed to the study design and data analysis; $\mathrm{XH}$, $\mathrm{FX}, \mathrm{ZW}, \mathrm{HY}, \mathrm{SQ}, \mathrm{XC}, \mathrm{CW}, \mathrm{HZ}, \mathrm{CL}$ contributed to data collection; ZW, HY, SQ, XC, CW, $\mathrm{HZ}, \mathrm{CL}, \mathrm{ZQ}$ were responsible for manuscript revision; and XH, QY, JH, FX contributed to manuscript writing.

Funding This work was supported by the Nanjing Municipal Medical Science and Technique Development Foundation, China (grant number 2007-ZKX07026).

Competing interests None declared.

Patient consent Obtained.

Ethics approval The study was approved by the academic and ethical committee of Nanjing Municipal Center for Disease Control and Prevention (Nanjing CDC).

Provenance and peer review Not commissioned; externally peer reviewed.

Data sharing statement № additional data are available.

Open access This is an open access article distributed in accordance with the Creative Commons Attribution Non Commercial (CC BY-NC 4.0) license, which permits others to distribute, remix, adapt, build upon this work non-commercially, and license their derivative works on different terms, provided the original work is properly cited and the use is non-commercial. See: http://creativecommons.org/ licenses/by-nc/4.0/

(C) Article author(s) (or their employer(s) unless otherwise stated in the text of the article) 2018. All rights reserved. No commercial use is permitted unless otherwise expressly granted.

\section{REFERENCES}

1. Stevens W, Peneva D, Li JZ, et al. Estimating the future burden of cardiovascular disease and the value of lipid and blood pressure control therapies in China. BMC Health Serv Res 2016;16:175. 
2. Wang W, Hu SS, Kong LZ, et al. Summary of report on cardiovascular diseases in China, 2012. Biomed Environ Sci 2014;27:552-8

3. Xu S, Jiayong Z, Li B, et al. Prevalence and clustering of cardiovascular disease risk factors among tibetan adults in china: a population-based study. PLoS One 2015;10:e0129966.

4. Yu J, Ma Y, Yang S, et al. Risk factors for cardiovascular disease and their clustering among adults in Jilin (China). Int J Environ Res Public Health 2015;13:70.

5. Gu D, Gupta A, Muntner P, et al. Prevalence of cardiovascular disease risk factor clustering among the adult population of China: results from the International Collaborative Study of Cardiovascular Disease in Asia (InterAsia). Circulation 2005;112:658-65.

6. Wu J, Cheng X, Quu L, et al. Prevalence and clustering of major cardiovascular risk factors in China: a recent cross-sectional survey. Medicine 2016;95:e2712.

7. Tao J, Ma YT, Xiang Y, et al. Prevalence of major cardiovascular risk factors and adverse risk profiles among three ethnic groups in the Xinjiang Uygur Autonomous Region, China. Lipids Health Dis 2013;12:185

8. Daviglus ML, Talavera GA, Avilés-Santa ML, et al. Prevalence of major cardiovascular risk factors and cardiovascular diseases among Hispanic/Latino individuals of diverse backgrounds in the United States. JAMA 2012;308:1775-84.

9. $\mathrm{Li} \mathrm{JH}$, Wang LM, Li YC, et al. Prevalence of major cardiovascular risk factors and cardiovascular disease in women in China: surveillance efforts. Biomed Environ Sci 2016;29:205-11.

10. Zhang L, Qin LQ, Cui HY, et al. Prevalence of cardiovascular risk factors clustering among suburban residents in Beijing, China. Int $J$ Cardiol 2011;151:46-9.

11. Wu DM, Pai L, Chu NF, et al. Prevalence and clustering of cardiovascular risk factors among healthy adults in a Chinese population: the MJ Health Screening Center Study in Taiwan. Int $J$ Obes Relat Metab Disord 2001:25:1189-95.

12. Gao B, Zhang L, Wang $\mathrm{H}$. Clustering of major cardiovascular risk factors and the association with unhealthy lifestyles in the chinese adult population. PLoS One 2013;8:e66780.

13. Yang HF, Chen XP, Hong X, et al. Influence of epidemiological trend of disease mortality on life expectancy among residents in Nanjing from 2011 to 2014. Jiangsu J Prev Med 2017;28:512-4. In Chinese.

14. Gupta S, Gudapati R, Gaurav K, et al. Emerging risk factors for cardiovascular diseases: Indian context. Indian J Endocrinol Metab 2013;17:806-14.

15. Sekhri T, Kanwar RS, Wilfred R, et al. Prevalence of risk factors for coronary artery disease in an urban Indian population. BMJ Open 2014;4:e005346.

16. Oluyombo R, Akinwusi PO, Olamoyegun $\mathrm{MO}$, et al. Clustering of cardiovascular risk factors in semi-urban communities in southwestern Nigeria. Cardiovasc J Afr 2016;27:322-7.

17. Park HS, Yun YS, Park JY, et al. Obesity, abdominal obesity, and clustering of cardiovascular risk factors in South Korea. Asia Pac J Clin Nutr 2003;12:411-8.

18. Selvarajah S, Haniff J, Kaur G, et al. Clustering of cardiovascular risk factors in a middle-income country: a call for urgency. Eur J Prev Cardiol 2013;20:368-75

19. Dai Y, Yuan BJ, Shi ZM. A cross-sectional study on diabetes mellitus among adults of 18 years old and above in Jiangsu Province. $J$ Hyg Res 2010;39:626-7. In Chinese.

20. Revision committee of Chinese guidelines for the management of hypertension. Chinese guidelines for the management of hypertension (Revised Edition 2014). Chin J Health Manage 2015;30:18-40. In Chinese.

21. Diabetes division of Chinese medical association. Chinese guidelines for the prevention and treatment of type 2 diabetes mellitus (2013 Version). Chin J Diabetes Mellitus 2014;30:26-89. In Chinese.

22. Revision committee of guidelines for prevention and treatment of dyslipidemia in Chinese adults. Guidelines for prevention and treatment of dyslipidemia in Chinese adults (Revised Edition 2016). Chinese Circulation Journal 2016;31:937-50. In Chinese.

23. Zhou BF. Cooperative Meta-Analysis Group of the Working Group on Obesity in China. Predictive values of body mass index and waist circumference for risk factors of certain related diseases in Chinese adults--study on optimal cut-off points of body mass index and waist circumference in Chinese adults. Biomed Environ Sci 2002;15:S685-93.

24. Yang G, Fan L, Tan J, et al. Smoking in China: findings of the 1996 National Prevalence Survey. JAMA 1999;282:1247-53.
25. Xu F, Yin XM, Wang Y. The association between amount of cigarettes smoked and overweight, central obesity among Chinese adults in Nanjing, China. Asia Pac J Clin Nutr 2007;16:240-7.

26. Xu F, Yin XM, Zhang $M$, et al. Family average income and body mass index above the healthy weight range among urban and rural residents in regional Mainland China. Public Health Nutr 2005:8:47-51.

27. Deng HB, Macfarlane DJ, Thomas GN, et al. Reliability and validity of the IPAQ-Chinese: the Guangzhou Biobank Cohort study. Med Sci Sports Exerc 2008;40:303-7.

28. US Department of Health and Human Services. Physical activity guidelines for Americans. 2008 http://www.health.gov/paguidelines (accessed on 20 May 2011).

29. Ye Q, Hong $X$, Wang Z, et al. Reproducibility and validity of an FFQ developed for adults in Nanjing, China. Br J Nutr 2016:115:887-94.

30. Ouyang X, Lou Q, Gu L, et al. Cardiovascular disease risk factors are highly prevalent in the office-working population of Nanjing in China. Int J Cardiol 2012;155:212-6.

31. Dukas L, Willett WC, Giovannucci EL. Association between physical activity, fiber intake, and other lifestyle variables and constipation in a study of women. Am J Gastroenterol 2003:98:1790-6.

32. Kerver JM, Yang EJ, Bianchi L, et al. Dietary patterns associated with risk factors for cardiovascular disease in healthy US adults. $A m \mathrm{~J}$ Clin Nutr 2003;78:1103-10.

33. Zhang L, Wang F, Wang L, et al. Prevalence of chronic kidney disease in China: a cross-sectional survey. Lancet 2012;379:815-22.

34. National Commission of Health Bureau of disease control and Prevention. Report on China national nutrition and chronic disease survey (2015). . Beijing: People's Medical Publishing House, 2015:11. 33-50.

35. Li N, Wang H, Yan Z, et al. Ethnic disparities in the clustering of risk factors for cardiovascular disease among the Kazakh, Uygur, Mongolian and Han populations of Xinjiang: a cross-sectional study. BMC Public Health 2012;12:499.

36. Pan L, Yang Z, Wu Y, et al. The prevalence, awareness, treatment and control of dyslipidemia among adults in China. Atherosclerosis 2016;248:2-9.

37. Li Q, Hsia J, Yang G. Prevalence of smoking in China in 2010. N Eng $J$ Med 2011;364:2469-70.

38. Stamler J, Stamler R, Neaton JD, et al. Low risk-factor profile and long-term cardiovascular and noncardiovascular mortality and life expectancy: findings for 5 large cohorts of young adult and middleaged men and women. JAMA 1999;282:2012-8.

39. Neaton JD. Serum cholesterol, blood pressure, cigarette smoking, and death from coronary heart disease overall findings and differences by age for 316099 white men. Arch Intern Med 1992;152:56-64.

40. Yusuf HR, Giles WH, Croft JB, et al. Impact of multiple risk factor profiles on determining cardiovascular disease risk. Prev Med 1998;27:1-9.

41. Yang ZJ, Liu J, Ge JP, et al. Prevalence of cardiovascular disease risk factor in the Chinese population: the 2007-2008 China National Diabetes and Metabolic Disorders Study. Eur Heart $J$ 2012;33:213-20.

42. Wang $X$, Yang F, Bots ML, et al. Prevalence and clustering of cardiovascular risk factors in adults in Northeast China. Heart Asia 2014;6:122-7.

43. Zhang B, Wang HJ, DU WW, et al. [Food consumption trend of Chinese adults in nine provinces (autonomous region) from 1989 to 2006]. Zhonghua Yu Fang Yi Xue Za Zhi 2011;45:330-4.

44. Ng SW, Howard AG, Wang HJ, et al. The physical activity transition among adults in China: 1991-2011. Obes Rev 2014;15(Suppl 1):27-36.

45. Chow CK, Jolly S, Rao-Melacini P, et al. Association of diet, exercise, and smoking modification with risk of early cardiovascular events after acute coronary syndromes. Circulation 2010;121:750-8.

46. EUROASPIRE II Study Group. Lifestyle and risk factor management and use of drug therapies in coronary patients from 15 countries; principal results from EUROASPIRE II Euro Heart Survey Programme. Eur Heart J 2001;22:554-72.

47. Nanjing Municipal Center for Disease Control and Prevention. Report on chronic disease risk factor surveillance in Nanjing, 2011. Nanjing: Jiangsu Science and Technology Press, 2014. In Chinese.

48. Liu J, Hong Y, D'Agostino RB, et al. Predictive value for the Chinese population of the Framingham $\mathrm{CHD}$ risk assessment too compared with the Chinese Multi-Provincial Cohort Study. JAMA 2004:291:2591-9. 